$11,12,19$

\title{
О критической точке плавления простого вещества
}

\author{
(C) М.Н. Магомедов \\ Институт проблем геотермии и возобновляемой энергетики - \\ филиал ФБГУН объединенного института высоких температур РАН, \\ Махачкала, Россия \\ E-mail: mahmag4@mail.ru
}

Поступила в Редакцию 25 декабря 2020 г. В окончательной редакции 25 декабря 2020 г. Принята к публикации 26 февраля 2021 г.

На основе трехфазной модели простого вещества изучен вопрос о появлении и исчезновении S-петли фазового перехода (ФП) первого рода на изотерме уравнения состояния в области ФП кристалл-жидкость (К-Ж). Расчеты, проведенные для аргона, показали, что S-петля ФП К-Ж на изотерме уравнении состояния возникает из-за резкого уменьшения и последующего роста давления, связанного с появлением делокализованных атомов при изотермическом увеличении удельного объема. С ростом температуры на изотерме давление, связанное с делокализацией атомов переходит из отрицательной области (где оно стягивало систему) в положительную область (где оно систему растягивает). Такое поведение данной функции приводит как к появлению S-петли ФП К-Ж на изотерме уравнения состояния, так и к исчезновению S-петли ФП К-Ж при высоких температурах с образованием критической точки ФП К-Ж. Изучено изменение параметров критической точки ФП К-Ж при уменьшении числа атомов в наносистеме. Показано, что при переходе к наносистеме критические температура и давление уменьшаются, а критический молярный объем возрастает. Расчеты в рамках трехфазной модели простого вещества показали, что структура в критической точке ФП К-Ж близка к аморфной упаковке. При этом с уменьшением числа атомов в наносистеме параметры данной аморфной структуры в критической точке ФП К-Ж меняются слабо.

Ключевые слова: плавление, уравнение состояния, критическая точка, аргон, нанокристалл.

DOI: $10.21883 /$ FTT.2021.07.51050.272

\section{1. Введение}

В 1873 г. Йоханнес Дидерик ван дер Ваальс (Johannes Diderik van der Waals) опубликовал свою теорию фазового перехода (ФП) первого рода для ФП жидкость-газ (Ж-Г). Эта теория объясняла, как появление S-петли ФП Ж-Г на изотерме уравнения состояния, так и исчезновение S-петли в критической точке ФП Ж-Г.

Сразу же после работы ван дер Ваальса возник вопрос о наличии критической точки у ФП первого рода кристалл-жидкость (К-Ж). История прогнозов и поисков критической точки ФП К-Ж подробно изложена в обзорах $[1,2]$. Дискуссия о критической точке ФП К-Ж прекратилась после статьи Ландау 1937 г. [3], [4, § 83]. В данной статье была доказана невозможность непрерывного перехода „между телами различной симметрии (в частности между жидкостью и кристаллом)“.

Однако это доказательство Ландау было подвергнуто сомнению Френкелем [5, с. 133-135], ввиду того, что в доказательстве Ландау не были учтены метастабильные и нестабильные состояния, которые присутствуют при ФП К-Ж. Френкель утверждал, что если учесть метастабильные и нестабильные состояния на изотерме уравнения состояния, то критическая точка для ФП К-Ж ,принципиально возможна“.

В последнее время применимость теоремы Ландау к жидкости и твердому телу при высоких давлениях $(P)$ и температурах $(T)$ также была поставлена под сомнение в работах [6], [7, с. 513], [8-14]. Дело в том, что при высоких $P-T$-условиях различие в структурах твердого тела и жидкости становится незначительным [15,16]. Кроме того, некоторые аргументы, доказывающие отсутствие критической точки ФП К-Ж имеют ряд недостатков. Например, в работе Стишова [6] экстраполяцией экспериментальных данных для скачков объема $\left(\Delta V / V_{s}\right)$ и энтропии $\left(\Delta s / k_{\mathrm{B}}\right.$, где $k_{\mathrm{B}}-$ постоянная Больцмана) аргона и натрия на значение $T=\infty$ были получены конечные величины

$$
\begin{aligned}
& \Delta V / V_{s} \rightarrow 0.03 \text { и } \Delta s / k_{\mathrm{B}} \rightarrow 0.9 \text { - для ГЦК-аргона, } \\
& \Delta V / V_{s} \rightarrow 0.0034 \text { - для ОЦК-натрия. }
\end{aligned}
$$

И хотя в [6] на основании этих экстраполяционных оценок было сделано утверждение об отсутствии критической точки ФП К-Ж, надо признать, что вопрос является открытым и сегодня, ибо данная экстраполяция неоднозначна. Кроме того, у натрия (как и у других щелочных металлов) на кривой плавления $\operatorname{Tm}(P)$ имеется максимум [17, Fig. 19], что делает экстраполяцию функции $\Delta V / V_{s}$ на значение $T=\infty$ не вполне корректной.

Вместе с тем, идея Ландау получила свое развитие в работе [18]. В этой работе было введено два параметра порядка. Первый параметр порядка - это плотность, а второй параметр порядка - упорядоченность. Первый параметр порядка - скалярный параметр 
порядка. Упорядоченность - это векторный параметр порядка. Данная модель исходит из гипотезы отсутствия критической точки ФП К-Ж, полагая что кристалл и жидкость различаются не только количественно, но и качественно - „внутренним порядком“. Именно поэтому в определение „параметра упорядоченности среды“ была введена ступенчатая функция Хевисайда от разности плотности среды и плотности в точке ФП К-Ж при заданной температуре. Легко понять, что такое приближение допустимо в окрестности тройной точки, но при высоких $P-T$-условиях (где различие в плотности и в структуре твердого тела и жидкости становится незначительным) использование ступенчатой функции становится не вполне корректным.

На сегодняшний день критическая точка ФП К-Ж экспериментально не обнаружена. Но поиски и прогнозы критической точки ФП К-Ж продолжаются [9-14]. В статье [9] на основе изложенных там соображений была сделана оценка $P-T$-параметров критической точки ФП К-Ж для железа

$$
T_{c m}=8632 \mathrm{~K}=8.632 \mathrm{kK}, P_{c m}=7.93 \mathrm{Mbar}=793 \mathrm{GPa} .
$$

В статье [11] на основе предложенной там аналитической модели была сделана оценка $P-T$-параметров критической точки ФП К-Ж для меди

$$
T_{c m}=184 \mathrm{kK}, \quad P_{c m}=102 \mathrm{Mbar}=10200 \mathrm{GPa} .
$$

Также в [11] были оценены значения $P_{c m}$ для других элементарных веществ. Было указано, что наименьшим значением $P_{c m}$ обладают наиболее сжимаемые элементарные кристаллы (гелий и щелочные металлы).

В работе [13] на основе трехфазной аналитической модели была сделана оценка $P-T-V$-параметров критической точки ФП К-Ж для аргона

$$
T_{c m}=17214 \mathrm{~K}=17.214 \mathrm{kK},
$$

$P_{c m}=388.24 \mathrm{kbar}=38.824 \mathrm{GPa}, V_{c r}=21.824 \mathrm{~cm}^{3} / \mathrm{mol}$.

Но на современном этапе указанные $P-T$-условия недостижимы в эксперименте. Возникает вопрос: при каких условиях можно экспериментально обнаружить критическую точку ФП К-Ж?

В данной работе на основе новой модификации трехфазной модели простого (однокомпонентного) вещества, которая была представлена в [19], был изучен вопрос о появлении и исчезновении S-петли ФП К-Ж на изотерме уравнения состояния аргона. Кроме того, было изучено изменение параметров критической точки ФП К-Ж при уменьшении числа атомов в наносистеме.

\section{2. Трехфазная модель простого вещества}

Будем полагать, что рассматриваемая система образует решеточную структуру из $N+N_{v}$ сферических ячеек одинакового объема, из которых $N_{v}$ ячеек вакантны, а $N$ ячеек заняты тождественными сферически симметричными атомами, масса каждого из которых равна $m$. Из $N$ атомов только часть атомов $\left(N-N_{d}\right)$ локализована в ячейках, а другая часть $\left(N_{d}\right)$ - делокализована. Если локализованные атомы колеблются в ячейках, то делокализованные атомы, приобретя в результате присущих статистической системе флуктуаций избыточную кинетическую энергию, перемещаться по всему объему системы $(V)$. Причем со временем локализованный атом, возбудившись, может стать делокализованным, и наоборот. Но если температура и плотность системы постоянны, то средняя (по системе) доля делокализованных атомов: $x_{d}=N_{d} / N$, как и средняя доля вакантных узлов: $\phi_{v}=N_{v} /\left(N+N_{v}\right)$, не изменяются.

Пусть атомы взаимодействуют посредством парного 4-х параметрического потенциала Ми-Леннард-Джонса, который имеет вид

$$
\varphi(r)=\frac{D}{(b-a)}\left[a\left(\frac{r_{o}}{r}\right)^{b}-b\left(\frac{r_{o}}{r}\right)^{a}\right] .
$$

где $D$ и $r_{o}$ - глубина и координата минимума потенциала, $b>a>1$ - численные параметры.

Если в решетке содержится $N_{v}$ вакансий, однородно распределенных по объему, то первое координационное число (т.е. число ближайших соседних атомов) равно

$$
k_{n}=\frac{k_{n}^{o} N}{N+N_{v}}=k_{n}^{o} \cdot\left(1-\phi_{v}\right),
$$

где $k_{n}^{o}$ - число ближайших к данному атому ячеек (как занятых, так и вакантных), т. е. это первое координационное число при $N_{v}=0, \phi_{v}-$ вероятность образования вакансии в решетке простого вещества [20]:

$$
\phi_{v}=\frac{N_{v}}{N+N_{v}}=\frac{2}{\pi^{1 / 2}} \int_{\left[E_{v} /\left(k_{\mathrm{B}} T\right)\right]^{1 / 2}}^{\infty} \exp \left(-t^{2}\right) \cdot d t,
$$

где $k_{\mathrm{B}}-$ постоянная Больцмана, $E_{v}-$ энергия создания вакантного узла в решетке.

Объем системы равен сумме объемов, приходящихся на одну (занятую либо вакантную) ячейку $\left(v_{a}\right)$, форму которой считаем сферической

$$
\begin{gathered}
V=\frac{\pi}{6 k_{p}}\left(N+N_{v}\right) c^{3}=\frac{v_{a}}{k_{p}} \frac{N}{\left(1-\phi_{v}\right)}, \\
v_{a}=\frac{\pi}{6} c^{3}=\frac{V}{N} k_{p}\left(1-\phi_{v}\right), \\
c_{o}=\left(\frac{6 k_{p} V}{\pi N}\right)^{1 / 3}=\left[\frac{6 v_{a}\left(\phi_{v}=0\right)}{\pi}\right]^{1 / 3},
\end{gathered}
$$

где $c=c_{o} \cdot\left(1-\phi_{v}\right)^{1 / 3}$ - расстояние между центрами ближайших ячеек, $k_{p}$ - коэффициент упаковки структуры из $\left(N+N_{v}\right)$ сферических ячеек, $c_{o}-$ расстояние между центрами ближайших ячеек в исходной (не срелаксировавшей в активированное вакансиями состояние) 
безвакансионной (при $\left.N_{v}=0\right)$ системе (на это указывает индекс , $\left.O^{\prime \prime}\right)$.

Очевидно, что центр делокализованного атома может перемещаться по всему объему системы, а центр локализованного атома только в пределах ячейки, в которой он локализован. Тогда для удельной (на атом) свободной энергии Гельмгольца макроскопической системы (где $N \rightarrow \infty$ и $V \rightarrow \infty$ при $N / V=$ const) можно получить выражение [19,21]:

$$
f_{H}=f_{i}+f_{s}+f_{w}+f_{d}+f_{k} .
$$

Здесь $f_{i}$ - это удельная свободная энергия трансляционного движения делокализованных атомов

$$
f_{i}=-x_{d} k_{\mathrm{B}} T\left[\left(\frac{T}{A_{d}}\right)^{3 / 2} \frac{V}{V_{0}}\right],
$$

где введены обозначения

$$
A_{d}=\left(\frac{2 \pi \hbar^{2}}{m k_{\mathrm{B}}}\right)\left(\frac{N}{e V_{0}}\right)^{2 / 3}, \quad V_{0}=N\left(\frac{\pi}{6 k_{p}}\right) r_{o}^{3} .
$$

Здесь $\hbar$ - постоянная Планка, $e=2.718$ - основание натурального логарифма, $x_{d}$ - доля делокализованных атомов, т. е. это вероятность атома иметь кинетическую энергию выше, чем $E_{d}$ - энергии делокализации атомa [22]:

$$
x_{d}=\frac{N_{d}}{N}=\frac{2}{\pi^{1 / 2}} \int_{E_{d} /\left(k_{\mathrm{B}} T\right)}^{\infty} t^{1 / 2} \exp (-t) \cdot d t .
$$

Второе слагаемое в (6) - это удельная свободная энергия статического взаимодействия всех атомов между собой, которая при использовании приближения „взаимодействия только ближайших соседей“ имеет вид

$$
f_{s}=\left(\frac{k_{n}^{o}}{2}\right)\left(1-\phi_{v}\right) D \cdot U_{o}(R)
$$

где $R=r_{o} / c-$ относительная линейная плотность системы,

$$
U_{o}(R)=\frac{a R^{b}-b R^{a}}{(b-a)}, \quad R=\frac{r_{o}}{c}=\left[\frac{V_{0}}{V\left(1-\phi_{v}\right)}\right]^{1 / 3} .
$$

Третье слагаемое в (6) - это удельная свободная энергия колебательного движения локализованных в ячейках атомов. В рамках модели колебательного спектра Эйнштейна данное слагаемое имеет вид $\left(\Theta_{E}-\right.$ температура Эйнштейна)

$$
f_{w}=3\left(1-x_{d}\right) k_{\mathrm{B}} T\left\{0.5 \frac{\Theta_{E}}{T}+\ln \left[1-\exp \left(-\frac{\Theta_{E}}{T}\right)\right]\right\} .
$$

Функция $f_{d}$ - это удельная свободная энергия динамического взаимодействия делокализованных атомов из-за их смещения от центров ячеек при миграции по объему. В приближении „взаимодействия только ближайших соседей“ для этой функции в работах [5, с. 216], [19], [23, с. 243] было получено выражение

$$
\begin{aligned}
f_{d}= & \frac{x_{d} k_{n}^{o}\left(1-\phi_{v}\right) a b D}{2(b-a)} \\
& \times\left\{R^{b}\left[\frac{l_{3}\left(b, \xi_{p}\right)}{b}\right]-R^{a}\left[\frac{l_{3}\left(a, \xi_{p}\right)}{a}\right]\right\},
\end{aligned}
$$

где введены обозначения

$$
\begin{gathered}
l_{3}(k, t)=\frac{(1+t)^{k-2}-(1-t)^{k-2}}{2(k-2) t\left(1-t^{2}\right)^{k-2}}-1, \\
\xi_{p}=\frac{0.5}{3^{1 / 2} k_{p}^{1 / 3}}<0.5 .
\end{gathered}
$$

Последнее слагаемое в (6) $f_{k}$ - это удельная свободная энергия „перестановочного движения“ атомов, которая возникает из-за применения формулы Стирлинга [19]:

$$
f_{k}=-\frac{k_{\mathrm{B}} T}{N} \ln \left[\frac{\left(N_{d} / e\right)^{N_{d}}}{N_{d} !}\right] \cong \frac{k_{\mathrm{B}} T}{2 N} \ln \left(2 \pi N_{d}\right) .
$$

Очевидно, что $f_{k}$ исчезает в „термодинамическом пределе“", т.е. при условии $N \rightarrow \infty, V \rightarrow \infty$ и $v=V / N$ $=$ const.

Входящие в (2)-(12) функции $E_{v}, E_{d}$ и $\Theta_{E}$ были определены для макросистемы простого вещества в работах $[20,21,24]$. Энергия создания вакансии в структуре однокомпонентного вещества имеет вид [20]:

$$
E_{v}=\frac{E_{L}}{1+x_{d}\left[\left(C_{D} E_{L} / k_{\mathrm{B}} T\right)-1\right]},
$$

где введены обозначения

$$
\begin{gathered}
E_{L}=\frac{m}{k_{n}^{o}}\left(\frac{c_{o} k_{\mathrm{B}} \Theta_{E_{0}}}{2 \hbar}\right)^{2} \cdot f_{y}\left(\frac{\Theta_{E_{0}}}{T}\right), \\
C_{D}=\frac{4 k_{n}^{o}}{3 k_{p}^{2 / 3}}, \quad f_{y}\left(y_{w}\right)=\frac{2}{y_{w}} \frac{\left[1-\exp \left(-y_{w}\right)\right]}{\left[1+\exp \left(-y_{w}\right)\right]} .
\end{gathered}
$$

Индекс „o“ у функций $c_{o}$ и $\Theta_{E_{0}}$ означает, что функции рассчитаны для исходной (не срелаксировавшей в активированное вакансиями состояние) безвакансионной виртуальной решетки (т.е. при $\left.\phi_{v}=0\right)$.

Для энергии делокализации атома было получено выражение [21,22]:

$$
\begin{aligned}
E_{d} & =\left(\frac{3}{8 \pi^{2}}\right) \cdot m \cdot\left(\frac{c_{o} k_{\mathrm{B}} \Theta_{E_{0}}}{\hbar k_{p}^{1 / 3}}\right)^{2} \cdot f_{y}\left(y_{w o}\right) \\
& =E_{d 1} \cdot f_{y}\left(y_{w o}\right)=C_{l d} \cdot E_{L},
\end{aligned}
$$

где введены обозначения

$$
\begin{gathered}
C_{l d}=\frac{3 k_{n}^{o}}{2 \pi^{2} k_{p}^{2 / 3}}=\left(\frac{9}{8 \pi^{2}}\right) C_{D}, \quad y_{w o}=\frac{\Theta_{E_{0}}}{T}, \\
E_{d 1}=E_{d}\left(f_{y}\left(y_{w o}\right)=1\right)=\frac{3 m}{8 k_{p}^{2 / 3}}\left(\frac{3 c_{o} k_{\mathrm{B}} \Theta_{o}}{4 \pi \hbar}\right)^{2} .
\end{gathered}
$$


Здесь $\Theta$ - температуры Дебая, которая связана с температурой Эйнштейна соотношением: $\Theta=(4 / 3) \Theta_{E}[23$, с. 796], $[25$, гл. 2, с. 116]. Для функции $\Theta$ было получено выражение следующего вида [24]:

$$
\Theta=A_{w} \xi\left[-1+\left(1+\frac{8 D}{k_{\mathrm{B}} A_{w} \xi^{2}}\right)^{1 / 2}\right],
$$

где введены следующие обозначения:

$$
\begin{gathered}
\xi=\frac{9}{k_{n}^{o}}, \quad A_{w}=K_{R} \frac{5 k_{n} a b(b+1)}{144(b-a)}\left(\frac{r_{o}}{c}\right)^{b+2}, \\
K_{R}=\frac{\hbar^{2}}{k_{\mathrm{B}} r_{o}^{2} m} .
\end{gathered}
$$

Было показано, что данные определения функций $E_{v}, E_{d}$ и $\Theta_{E}$ позволяют получить результаты, хорошо согласующиеся с экспериментальными оценками. Кроме этого, использование входящих в (6) функций $E_{v}, E_{d}$ и $\Theta_{E}$ в виде $(15)-(20)$ приводит к выполнимости как третьего начала термодинамики в „сильной“ формулировке Планка, так и к согласованности термического и калорического уравнений состояния для макросистемы. Было показано, что данный формализм полностью учитывает „коллективную энтропию“ (communal entropy) [6], [23, с. 227], [26]. „Коллективная энтропия“ определяет собой ту поправку, которую необходимо было включать в „твердоподобные“ или в „газоподобные“ теории жидкого состояния из-за некорректного учета в них как разницы в областях доступности у локализованных и делокализованных атомов, так и для корректного учета числа возможных перестановок в системе. В соответствии с (14), „параметр коллективной энтропии“ равен: $\sigma_{c o m}=\exp \left[x_{d}(v, T)\right]$. Отсюда при $x_{d}=1$ (т. е. для газа при высоких $T$ ) получаем: $\sigma_{c o m}=e$, а при $x_{d}=0$ (т.е. для кристалла при низких $T$ ) имеем: $\sigma_{\text {com }}=1$.

Данная решеточная модель основана на идеях Я.И. Френкеля [5, гл. 3 и 4], который указал, что кристаллическое и жидкое состояния однокомпанентного вещества различаются только долей вакантных узлов в виртуальной решетке, и долей подвижных атомов. В нашем подходе эти функции $\phi_{v}$ и $x_{d}$ связаны между собой через энергетические параметры (15)-(17).

\section{3. Расчет изотерм уравнения состояния для макросистемы}

Для расчетов был взят аргон $(m(\mathrm{Ar})=39.95$ a.m.u. $)$, имеющий в твердой фазе гранецентрированную кубическую (ГЦК) структуру: $k_{n}^{o}=12, k_{p}=0.7405$. В связи с этим, структурные параметры решеточной модели будут равны

$$
\begin{gathered}
C_{D}=4 k_{n}^{o} /\left(3 k_{p}^{2 / 3}\right)=19.548 \text { из }(16), \\
C_{l d}=3 k_{n}^{o} /\left(2 \pi^{2} k_{p}^{2 / 3}\right)=2.2282 \text { из }(18), \\
\xi=9 / k_{n}^{o}=0.75 \text { из }(20) .
\end{gathered}
$$

Параметры парного межатомного потенциала МиЛеннард-Джонса (1) для Ar были определены самосогласованным образом из экспериментальных данных твердой фазы в [27]:

$$
\begin{gathered}
r_{o}=3.7555 \cdot 10^{-10} \mathrm{~m}, \quad D / k_{\mathrm{B}}=173.6 \mathrm{~K}, \\
a=6, \quad b=17 .
\end{gathered}
$$

При использовании параметров из (21) входящие в формализм термодинамические константы модели будут равны

$$
\begin{gathered}
v_{o}=\left(\pi / 6 k_{p}\right) r_{o}^{3}=37.4522 \cdot 10^{-30} \mathrm{~m}^{3}, \\
V_{o}=v_{o} N_{A}=22.5542 \mathrm{~cm}^{3} / \mathrm{mol}, \Theta_{E}(1)=65.106 \mathrm{~K} \\
\Theta(1)=(4 / 3) \Theta_{E}(1)=86.807 \mathrm{~K}, \quad \gamma(1)=3.011
\end{gathered}
$$

Здесь $N_{A}=6.02214 \cdot 10^{23} \mathrm{~mol}^{-1}$ - число Авогадро (Avogadro constant), $\gamma=-(\partial \ln \Theta / \partial \ln v)_{T}$ - первый параметр Грюнайзена. Значение 1 у функций $A_{w}, \Theta_{E}$ и $\gamma$ указывает на то, что они определены при аргументе равном: $R=r_{o} / c=1$.

Расчет нормированного термического уравнения состояния проводился численным дифференцированием изотермической зависимости функции $f_{H} / D$ по аргументу $v / v_{o}=\left(c_{o} / r_{o}\right)^{3}$, т. е. по формуле

$$
\frac{P v_{o}}{D}=-\left[\frac{\partial\left(f_{H} / D\right)}{\partial\left(v / v_{o}\right)}\right]_{T} .
$$

Здесь $v=V / N=\left(\pi / 6 k_{p}\right) c_{o}^{3}$ - удельный (на атом) объем системы при расстоянии между центрами ближайших атомов равном $c_{o}$.

Расчет изотермической зависимости функции $P v_{o} / D$ от аргумента $v / v_{o}$ показал [19], что при средних температурах изотермы имеют две $\mathrm{S}$-петли, соответствующие по физическому смыслу ФП К-Ж и Ж-Г. При высоких температурах S-петля ФП Ж-Г стягивается в критическую точку. При низких температурах две S-петли ФП К-Ж и Ж-Г сливаются в одну большую S-петлю, соответствующую ФП кристалл-газ. Таким образом, предложенная модель позволяет с единых позиций получить качественную картину для всех трех фаз простого вещества. При этом в модели не содержится никаких „внешних критериев“ для запуска ФП К-Ж (типа критерия Линдеманна $[1,2])$, и никаких подгоночных постоянных. Но количественные результаты данной модели еще далеки от экспериментальных данных. Например, для критической точки ФП Ж-Г в [19] были получены следующие параметры:

$$
\begin{gathered}
T_{c r}=249 \mathrm{~K}, V_{c r}=71.98 \mathrm{~cm}^{3} / \mathrm{mol}, P_{c r}=0.016215 \mathrm{GPa}, \\
Z_{c r}=\left[P v /\left(k_{\mathrm{B}} T\right)\right]_{c r}=0.564, v_{c r} / v_{o}=3.1915, \\
P_{c r} v_{o} / D=0.2534, \\
\phi_{v c r}=0.750138, k_{n c r}=2.99835, x_{d c r}=0.998754 .
\end{gathered}
$$


Значения из (22) существенно отличаются от экспериментальных параметров критической точки ФП Ж-Г аргона, которые равны [28]:

$$
\begin{gathered}
T_{c r}=150.9 \mathrm{~K}, \quad V_{c r}=74.6 \mathrm{~cm}^{3} / \mathrm{mol}, \\
P_{c r}=0.004777 \mathrm{GPa}, \quad Z_{c r}=0.290 .
\end{gathered}
$$

Экспериментальное значение первого координационного числа в критической точке ФП Ж-Г аргона равно: $k_{n c r}=3.6 \pm 0.1=3.5-3.7$ [29]. Согласно (3), вероятность образования вакантного узла равна: $\phi_{v}=1-\left(k_{n} / k_{n}^{o}\right)=1-\left(k_{n} / 12\right)$, Отсюда для критической точки ФП Ж-Г получим: $\phi_{v c r}=0.7 \pm 0.0083$ [29]. Это достаточно близко к полученным нами величинам $\phi_{v \text { cr }}$ и $k_{n c r}$ из (22).

Представленный здесь формализм трехфазной модели можно использовать для изучения качественных вопросов природы ФП К-Ж. В данном случае на основе этой модели был изучен вопрос появления критической точки для S-петли ФП К-Ж при высоких $P-T$-условиях, а также было изучено изменение параметров критической точки ФП К-Ж при уменьшении размера наносистемы.

На рис. 1 показаны изотермы уравнения состояния в области S-петли ФП К-Ж при $T=15-20 \mathrm{kK}$. Из рис. 1 видно, что в данной трехфазной модели аргона для ФП К-Ж имеется критическая точка со следующими параметрами:

$$
\begin{gathered}
T_{c m}=17.87 \mathrm{kK}, \quad V_{c m}=11.575 \mathrm{~cm}^{3} / \mathrm{mol}, \\
P_{c m}=249.5 \mathrm{GPa}, Z_{c m}=\left[P v /\left(k_{\mathrm{B}} T\right)\right]_{c m}=37.872, \\
v_{c m} / v_{o}=0.5132, \quad P_{c m} v_{o} / D=3898.5, \\
\phi_{v c m}=0.43562, \quad k_{n c m}=6.7725, \quad x_{d c m}=0.13302 .
\end{gathered}
$$

Полученное значение $k_{n c m}$ из (23) указывает на то, что структура в критической точке ФП К-Ж близка к аморфной упаковке, для которой другими методами получены следующие оценки: $k_{\text {namorph }}=6-6.08$ [30], 6.2793 [31]. Именно близость структур твердой и жидкой фаз при высоких $P-T$-условиях и позволяет реализоваться критической точке ФП К-Ж не нарушая при этом теорему Л.Д. Ландау $[3,4]$.

Отметим, что вышеуказанные результаты получены нами в предположении, что параметры парного межатомного потенциала из (21) не изменяются вплоть до указанных в (23) $P-T$-условий. Если же параметры межатомного потенциала в силу различных причин будут изменяться, то представленные в (23) значения критической точки ФП К-Ж могут быть иными.

В соответствии с (6), уравнение состояния системы можно представить в виде

$$
P=P_{s}+P_{i}+P_{w}+P_{d}=P_{s w}+P_{i d} .
$$

Здесь $P_{s}=-\left(\partial f_{s} / \partial v\right)_{T}-$ давление из-за статического взаимодействия всех атомов между собой,

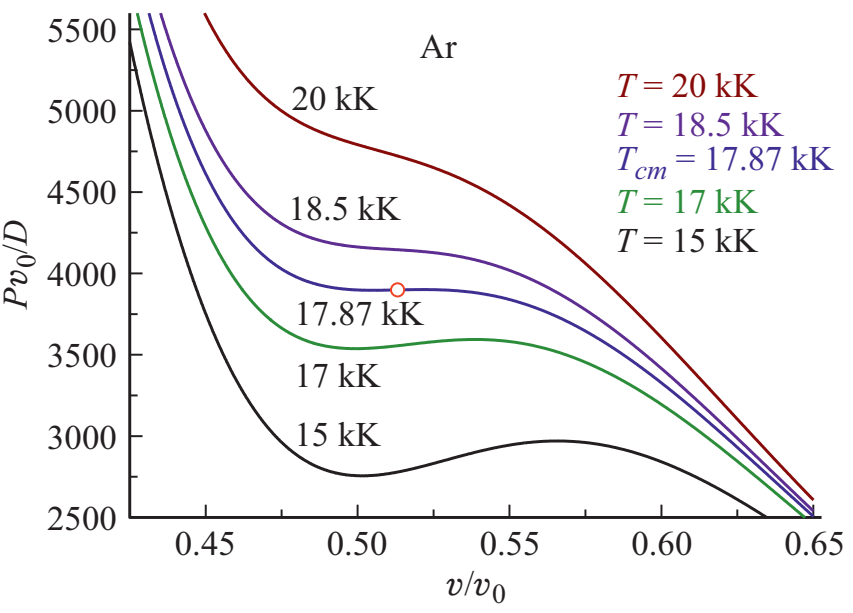

Рис. 1. Изотермы уравнения состояния в области S-петли ФП К-Ж при $T=15-20 \mathrm{kK}$.

$P_{i}=-\left(\partial f_{i} / \partial v\right)_{T}$ - давление от трансляционного движения делокализованных атомов, $P_{w}=-\left(\partial f_{w} / \partial v\right)_{T}-$ давление из-за колебательного движения локализованных атомов, $P_{d}=-\left(\partial f_{d} / \partial v\right)_{T}-$ давление из-за динамического взаимодействия делокализованных атомов со своим окружением при их смещении от центров ячеек в процессе миграции по объему, $P_{s w}=P_{s}+P_{w}$, $P_{i d}=P_{i}+P_{d}$.

Из формулы (6) легко видеть, что при $x_{d}=0$ получим: $f_{H}\left(x_{d}=0\right)=f_{s}+f_{w}$, т.е. это удельная свободная энергия кристалла при низких температурах. При $x_{d}=1$ и при значении $\phi_{v}$ близком к единице из (6) получим удельную свободную энергию газа: $f_{H}\left(x_{d}=1\right)=f_{i}+f_{s}+f_{d}$. Поэтому величину $P_{s w}=P_{s}+P_{w}$ из (24) можно назвать давлением от „твердоподобных“ атомов, а $P_{i d}=P_{i}+P_{d}$ - давлением от „газоподобных“ атомов.

На рис. 2 показаны изотермы общего давления (сплошная толстая линия) и вкладов, которые его составляют в (24), как для $T=20 \mathrm{kK}$ (верхний график), так и для $T=15 \mathrm{kK}$ (средний график). На нижнем графике рис. 2 показаны изотермы общего давления (сплошные толстые линии) при $T=20$ (верхняя линия) и 15 (нижняя линия) $\mathrm{kK}$, и составляющих его двух вкладов: от „твердоподобных“ и „газоподобных“ атомов: $P_{s w}$ - тонкие линии с минимумом, $P_{i d}$ - тонкие линии с минимумом и максимумом. Тонкие штриховые линии относятся к $T=15 \mathrm{kK}$, тонкие пунктирные линии к $T=20 \mathrm{kK}$.

Из рис. 2 легко понять, что S-петля ФП К-Ж на изотерме уравнении состояния возникает из-за резкого уменьшения и последующего роста давления, связанного с появлением делокализованных атомов при изотермическом увеличении удельного объема. При этом, если на изотерме $T=15 \mathrm{kK}$ в предплавительной области функции $P_{d}$ и $P_{i d}$ отрицательные (т.е. они стягивают кристалл), то на изотерме $T=20 \mathrm{kK}$ эти функции стано- 
вятся положительными (т. е. они систему растягивают). Такое поведение данных функций обуславливает как появление $\mathrm{S}$-петля ФП К-Ж на изотерме уравнении
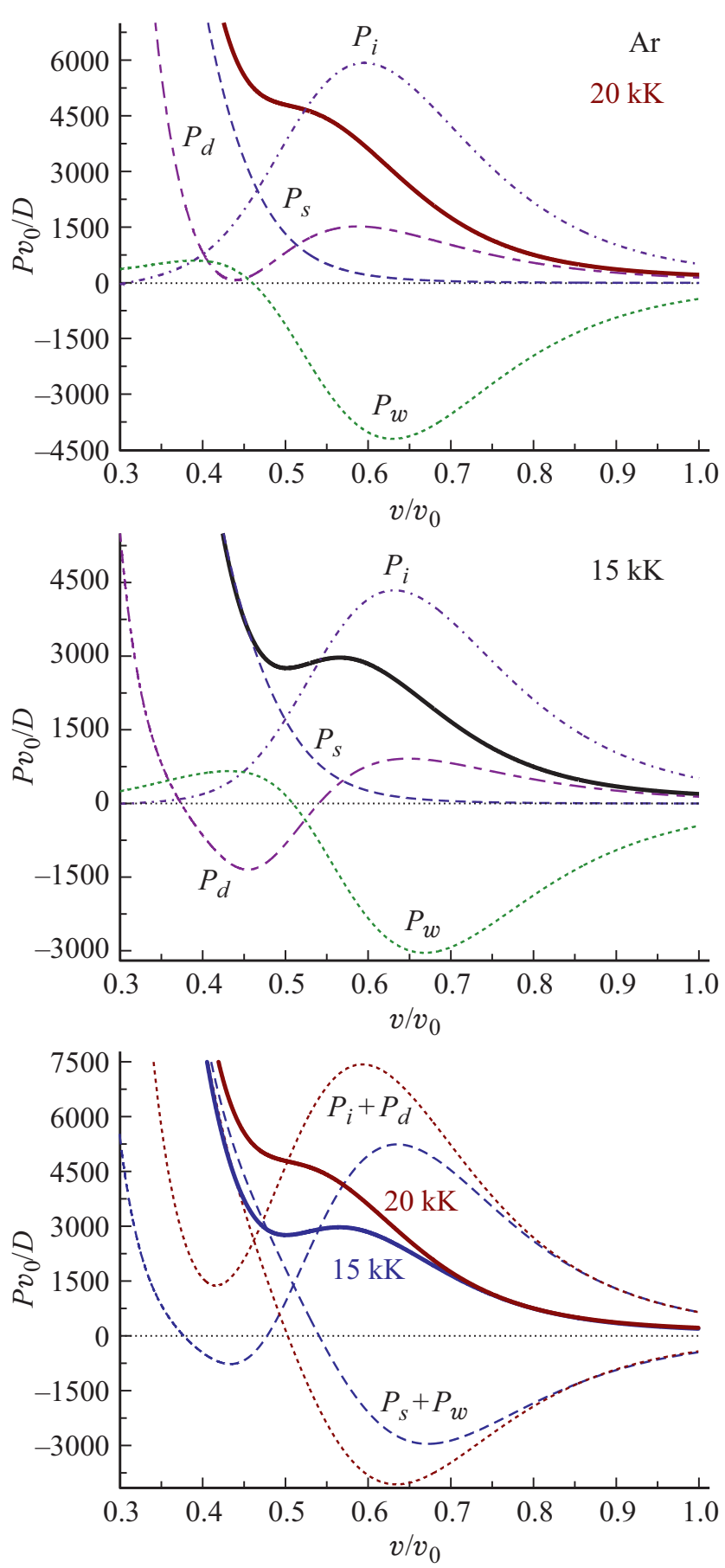

Рис. 2. Изотермы общего давления (сплошная толстая линия) и его составляющих при $T=20$ (верхний график) и 15 (средний график) kK: $P_{s}$ - штриховая линия, $P_{i}$ - штрихпунктирная линия, $P_{w}-$ пунктирная линия, $P_{d}-$ штрихпунктирная линия. На нижнем графике показаны изотермы общего давления (сплошные толстые линии), и составляющих его двух вкладов: $P_{s w}=P_{s}+P_{w}-$ тонкие линии с минимумом и $P_{i d}=P_{i}+P_{d}-$ тонкие линии с минимумом и максимумом. Штриховые линии относятся к $T=15 \mathrm{kK}$, пунктирные линии - к $T=20 \mathrm{kK}$. состояния, так и ее исчезновение в критической точке ФП К-Ж при высоких температурах.

Но полученные для макросистемы аргона $P-T$-параметры критической точки ФП К-Ж очень высокие и недостижимы для современных экспериментов. Ввиду этого нами была изучена эволюция параметров критической точки ФП К-Ж при уменьшении числа атомов в системе. При этом нами была использована RP(vac)модель нанокристалла, представленная в работах $[19,32]$.

\section{4. Модель нанокристалла с вакансиями и самодиффузией}

Обобщим формализм из (2)-(20) на случай нанокристалла из $N+N_{v}=N /\left(1-\phi_{v}\right)$ одинаковых ячеек, из которых $N_{v}$ вакантных ячеек однородно распределено по объему нанокристалла. Как и в работах $[19,32]$ положим, что нанокристалл со свободной поверхностью имеет вид прямоугольного параллелепипеда с квадратным основанием, ограненный гранями типа (100) с геометрической поверхностью Гиббса. Ячейки образуют кристаллическую структуру с коэффициентом упаковки $k_{p}$. Величина $f=N_{p s} / N_{p o}=N_{p s}^{o} / N_{p o}^{o}$ - представляет собой параметр формы, который определяется отношением числа $N_{p s}^{o}$ атомов (или $N_{p s}=N_{p s}^{o} /\left(1-\phi_{v}\right)^{1 / 3}$ ячеек) на боковом ребре к числу $N_{p o}^{o}$ атомов (или $N_{p o}=N_{p o}^{o} /\left(1-\phi_{v}\right)^{1 / 3}$ ячеек) на ребре квадратного основания. Для стержневидной формы $f>1$, для куба $f=1$, для пластинчатой формы $f<1$.

Число ячеек и атомов в нанокристалле определенной формы (т. е. при данном $f$ ) равно

$$
\begin{gathered}
N+N_{v}=\operatorname{INT}\left(f \frac{N_{p o}^{3}}{\alpha}\right)=\operatorname{INT}\left[f \frac{\left(N_{p o}^{o}\right)^{3}}{\alpha\left(1-\phi_{v}\right)}\right], \\
N=\operatorname{INT}\left[f \frac{\left(N_{p o}^{o}\right)^{3}}{\alpha}\right],
\end{gathered}
$$

где $\alpha=\pi /\left(6 k_{p}\right)$ - параметр структуры, функция INT $[x]$ округляет величину $x$ до целого значения, ибо число ячеек и атомов это величины целочисленные.

Ограничение системы поверхностью приводит к обрыву связей на границе. Поэтому если использовано приближение „взаимодействия только ближайших соседей“, то вместо первого координационного числа $\left(k_{n}\right)$ необходимо брать $\left\langle k_{n}\right\rangle$ - среднее (по всей наносистеме) значение первого координационного числа, которое будет зависеть как от размера, так и от формы наносистемы. При этом структуру системы полагаем неизменной: $k_{p}=$ const. Данную модель нанокристалла в виде прямоугольного параллелепипеда (Rectangular Parallelepiped), форму которого можно варьировать с помощью параметра формы $f$, была названа RP-моделью с вакансиями, или RP(vac)-моделью [32].

B рамках $\mathrm{RP}(\mathrm{vac})$-модели зависимость среднего по наносистеме значения первого координационного числа 
от аргументов $\alpha, N, \phi_{v}$ и $f$ имеет вид [32]:

$$
\begin{aligned}
k_{n}\left(N, \phi_{v}\right)= & k_{n}^{o}(\infty) \cdot\left(1-\phi_{v}\right) \\
& \times\left[1-Z_{s}(f) \cdot\left(\frac{\alpha^{2}}{N}\left(1-\phi_{n}\right)\right)^{1 / 3}\right],
\end{aligned}
$$

где $k_{n}^{o}(\infty)$ - первое координационное число (т. е. число ближайших к данному атому занятых или вакантных ячеек) для макрокристалла,

$$
Z_{s}(f)=\frac{1+2 f}{3 f^{2 / 3}} .
$$

Функция формы $Z_{s}(f)$ достигает минимума равного единице при $f=1$, т. е. для формы куба. Для пластинчатых $(f<1)$ или стержневидных $(f>1)$ форм значение функции формы больше единицы: $Z_{s}(f \neq 1)>1$. Поэтому функция $k_{n}(f)^{*}=k_{n}(f) / k_{n}(\infty)$ при любом значении $N+N_{v}$ имеет максимум при $f=1$, т.е. для наиболее энергетически оптимальной - кубической формы прямоугольного параллелепипеда.

Кубическая форма может реализовываться только при определенном числе ячеек, из которого можно построить куб: $\left(N+N_{v}\right)_{\mathrm{cub}}=\operatorname{INT}\left[\left(N_{p o}^{o}\right) 3 /\left[\alpha\left(1-\phi_{v}\right)\right]\right]$, где $N_{p o}^{o}=2,3,4, \ldots$. При „некубичном“ значении числа ячеек: $N+N_{v} \neq\left(N+N_{v}\right)_{\text {cub }}$ параллелепипед может иметь либо пластинчатую, либо стержневидную форму, причем выполняется:

$$
k_{n}\left(\left(N+N_{v}\right)_{\mathrm{cub}} \pm 1\right)^{*}<k_{n}\left(N+N_{v}\right)_{\mathrm{cub}}^{*}
$$

Для наносистемы со свободной поверхностью Гиббса делокализация атома может происходить двумя различными путями: внутри объема и на поверхности Гиббса. Допустим, что делокализация атома со свободной поверхности Гиббса связана с переходом атома в газовую фазу и возвратом атома (этого либо другого) обратно. Тогда для доли числа делокализованных атомов в наносистеме получим [19]:

$$
x_{d}=\frac{\left(N-N_{d s}\right) x_{d v}+N_{d s} x_{d s}}{N}=\left(1-\eta_{d s}\right) x_{d v}+\eta_{d s} x_{d s},
$$

Здесь $x_{d v}$ - доля делокализованых атомов в объеме, которая рассчитывается по формулам (9) и (17), $x_{d s}-$ это вероятность делокализации атома на поверхности Гиббса способом, отличным от того, каким атом делокализуется в объеме. Как было показано в [19], для делокализация атома со свободной поверхности Гиббса в газовую фазу и возвратом атома обратно, можно принять: $x_{d s}=0.5$.

Доля атомов, делокализованных на поверхности Гиббса по способу, отличному от „объемного“ способа, равна [19]:

$$
\begin{aligned}
\eta_{d s} & =\frac{N_{d s}}{N}=\frac{2(2 f+2)}{3 f N_{p o}^{o}}\left(1-\phi_{v}\right)^{1 / 3}\left(1-x_{d v}\right) \\
& =2 \frac{Z_{s}(f)}{(\alpha N)^{1 / 3}}\left(1-\phi_{v}\right)^{1 / 3}\left(1-x_{d v}\right) .
\end{aligned}
$$

Из (29) видно, что для идеального $\left(x_{d v}=0\right.$ и $\left.\phi_{v}=0\right)$ нанокристалла функция $\eta_{d s}(N)$ плавно убывает от $\eta_{d s}=1$ при $N_{\min }=11$ и $Z_{s}(f=1)=1$ (или $N_{p o}^{o}=2$ и $f=1)$ до нуля при $N \rightarrow \infty$.

Таким образом, использование функций (25)-(29) в формализме $(2)-(20)$ при $k_{p}=$ const позволяет получить зависимость свободной энергии Гельмгольца, как от размера (числа атомов $N$ ), так и от формы нанокристалла $(f)$ при данных значениях температуры и удельного объема $(v=V / N)$, для нанокристалла со свободной геометрической поверхностью Гиббса.

\section{5. Изменение параметров критической точки для нано-системы}

Изучения S-петли ФП К-Ж на изотермах уравнения состояния аргона были проведены для наносистемы кубической формы (т.е. при $f=1$ ) при трех значениях числа атомов:

$$
N_{p o}^{o}=100,
$$

т. е. $N=\operatorname{INT}\left[(f=1)\left(N_{p o}^{o}\right)^{3} /(\alpha=0.70709)\right]=1414248$;

$N_{p o}^{o}=50$, т.е. $N=176781 ; N_{p o}^{o}=20$, т. е. $N=11314$.

Расчет изотермо-изоморфных (т.е. при постоянных значениях $T$ и $f$ ) зависимостей уравнения состояния показал, что при уменьшении числа атомов $P-V-T$ параметры критической точки ФП К-Ж изменяются: критические температура $T_{c m}$ и давление $P_{c m}$ уменьшаются, а критический молярный объем $V_{c m}$ возрастает. Результаты расчетов представлены в таблице.

Как видно из таблицы, в критической точке ФП К-Ж некоторые функции можно полагать независящими от

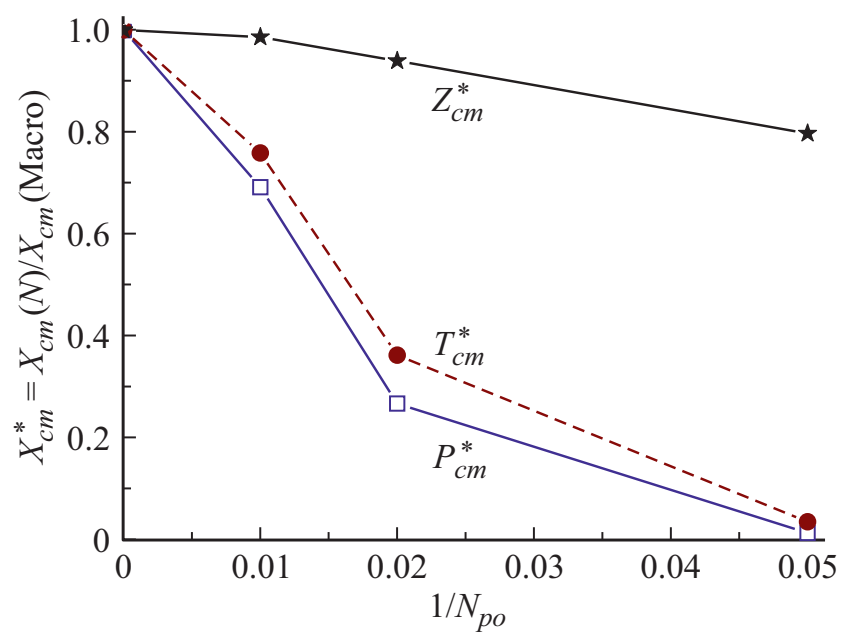

Рис. 3. Зависимости от $1 / N_{p o}$ нормированных значений температуры $T_{c m}^{*}$ (штриховая линия с точками), давления $P_{c m}^{*}$ (сплошная линия с квадратами) и величины $Z_{c m}^{*}$ (сплошная линия со звездочками) в критической точке ФП К-Ж для наносистемы Ar. 
Параметры критической точки ФП К-Ж при различном числе атомов в наносистеме кубической формы

\begin{tabular}{l|c|c|c|c}
\hline \multicolumn{1}{c|}{$\begin{array}{c}N_{p o} \\
N\end{array}$} & $\begin{array}{c}\text { Macro } \\
N=\infty\end{array}$ & $\begin{array}{c}N_{p o}=100 \\
N=1414248\end{array}$ & $\begin{array}{c}N_{p o}=50 \\
N=176781\end{array}$ & $\begin{array}{c}N_{p o}=20 \\
N=11314\end{array}$ \\
\hline$T, \mathrm{~K}$ & 17870 & 13560 & 6480 & 645 \\
\hline$P, \mathrm{GPa}$ & 249.5 & 172.6 & 66.8 & 3.55 \\
\hline$V, \mathrm{~cm}^{3} / \mathrm{mol}$ & 11.575 & 12.522 & 14.723 & 23.411 \\
\hline$Z=P v /\left(k_{\mathrm{B}} T\right)$ & 19.436 & 19.166 & 18.256 & 15.497 \\
\hline$v / v_{o}$ & 0.5132 & 0.5552 & 0.6528 & 1.0380 \\
\hline$c_{o} / r_{o}$ & 0.8006 & 0.8219 & 0.8675 & 1.0125 \\
\hline$c\left(\phi_{v}\right) / r_{o}$ & 0.6615 & 0.6787 & 0.7292 & 0.8410 \\
\hline$\Theta\left(\phi_{v}\right), \mathrm{K}$ & 419.16 & 347.52 & 233.17 & 58.50 \\
\hline$\gamma\left(\phi_{v}\right)$ & 2.240 & 2.435 & 2.711 & 3.063 \\
\hline$q\left(\phi_{v}\right)^{1}$ & 1.198 & 0.901 & 0.522 & 0.107 \\
\hline$E_{d} /\left(k_{\mathrm{B}} T\right)$ & 2.7979 & 2.7804 & 2.9539 & 2.8002 \\
\hline$x_{d}=N_{d} / N$ & 0.1330 & 0.1403 & 0.1276 & 0.1592 \\
\hline$E_{v} /\left(k_{\mathrm{B}} T\right)$ & 0.3039 & 0.3022 & 0.3453 & 0.3155 \\
\hline$\phi_{v}=N_{v} /\left(N+N_{v}\right)$ & 0.4356 & 0.4369 & 0.4060 & 0.4270 \\
\hline $\lg \left(\phi_{v} / x_{d}\right)$ & 0.5152 & 0.4935 & 0.5027 & 0.4284 \\
\hline$k_{n}\left(\phi_{v}\right)^{*}$ & 0.5644 & 0.5598 & 0.5870 & 0.5562 \\
\hline$k_{n}\left(\phi_{v}\right)$ & 6.77 & 6.72 & 7.04 & 6.67
\end{tabular}

Примеч ан ие. ${ }^{1}$ Здесь $q=(\partial \ln \gamma / \partial \ln v)_{T}-$ второй параметр Грюнайзена.

размера системы. Например, можно принять

$$
\begin{gathered}
E_{d} /\left(k_{\mathrm{B}} T\right)=2.8, x_{d}=N_{d} / N=0.14, E_{v} /\left(k_{\mathrm{B}} T\right)=0.32, \\
\phi_{v}=N_{v} /\left(N+N_{v}\right)=0.43, \lg \left(\phi_{v} / x_{d}\right)=0.5, \\
k_{n}\left(\phi_{v}\right)^{*}=0.56, \quad k_{n}\left(\phi_{v}\right)=6.7 .
\end{gathered}
$$

На рис. 3 показаны зависимости от $1 / N_{p o}$ нормированных значений температуры $T_{c m}^{*}$, давления $P_{c m}^{*}$ и величины $Z_{c m}^{*}$ в критической точке ФП К-Ж для наносистемы Ar. Здесь $X_{c m}^{*}=X_{c m}(N) / X_{c m}($ Macro $)$ - значение критического параметра для наносистемы нормированное на соответствующее значение для критической точки ФП К-Ж в макросистеме.

Если форма наносистемы со свободной поверхностью Гиббса будет отлична от энергетически оптимальной формы (для параллелепипеда это форма куба), то размерные зависимости функций $T_{c m}^{*}, P_{c m}^{*}$ и $Z_{c m}^{*}$ будут заметнее ввиду более сильной зависимости функций $k_{n}(N)$ и $\eta_{d s}(N)$ при $f \neq 1$ в формулах (26) и (29), соответственно.

Если наносистема с данной формой поверхности одной гранью лежит на подложке, то число атомов на свободной поверхности Гиббса (т.е. величина $N_{d s}$ в (29)) уменьшается. Это приводит к тому, что размерные зависимости функций $T_{c m}^{*}, P_{c m}^{*}$ и $Z_{c m}^{*}$ становятся меньше, чем для безопорного нанокристалла.

Таким образом, изучая плавление нанокристаллов инертных газов (или других веществ с низкими температурами плавления) можно обнаружить критическую точку ФП К-Ж при относительно низких $P-T$-условиях.

Отметим, что, как было показано в работах [19,33,34], $P-V-T$-параметры критической точки ФП Ж-Г при переходе к наносистеме изменяются аналогичным образом: критические температура и давление уменьшаются, а критический молярный объем возрастает.

\section{6. Заключение}

На основе трехфазной модели простого вещества изучен вопрос о появлении и исчезновении $\mathrm{S}$-петли ФП К-Ж на изотерме уравнения состояния аргона. Показано, что S-петля ФП К-Ж на изотерме уравнении состояния возникает из-за резкого уменьшения и последующего роста давления, связанного с появлением делокализованных атомов при изотермическом увеличении удельного объема. С ростом температуры на изотерме давление, связанное с делокализацией атомов переходит из отрицательной области (где оно стягивало систему) в положительную область (где оно систему растягивает). Такое поведение данной функции обуславливает как появление S-петля ФП К-Ж на изотерме уравнении состояния, так и исчезновение S-петля ФП К-Ж при высоких температурах, с появлением критической точки ФП К-Ж.

B рамках $\mathrm{RP}(\mathrm{vac})$-модели изучено изменение параметров критической точки ФП К-Ж при уменьшении числа атомов в наносистеме. Показано, что при переходе к наносистеме критические температура и давление уменьшаются, а критический молярный объем возрастает.

Расчеты в рамках представленной трехфазной модели простого вещества показали, что структура в критической точке ФП К-Ж близка к аморфной упаковке. При этом, с уменьшением числа атомов в наносистеме параметры данной аморфной структуры меняются слабо.

Показано, что для наносистемы с формой свободной поверхности Гиббса отличной от энергетически оптимальной формы, размерные зависимости функций $T_{c m}^{*}$, $P_{c m}^{*}$ и $Z_{c m}^{*}$ будут заметнее. Если наносистема с данной формой поверхности одной гранью лежит на подложке, то размерные зависимости функций $T_{c m}^{*}, P_{c m}^{*}$ и $Z_{c m}^{*}$ становятся слабее.

\section{Благодарности}

Автор выражает благодарность С.П. Крамынину, Н.Ш. Газановой, 3.М. Сурхаевой и М.М. Гаджиевой за плодотворные дискуссии и помощь в работе. 


\section{Финансирование работы}

Работа выполнена при финансовой поддержке РФФИ (грант № 18-29-11013_мк).

\section{Конфликт интересов}

Автор заявляет, что у него нет конфликта интересов.

\section{Список литературы}

[1] А. Уббелоде. Плавление и кристаллическая структура. Мир, М. (1969). 420 c. [A.R. Ubbelohde. Melting and Crystal Structure. Clarendon Press, Oxford, U.K. (1965)].

[2] J. Bilgram. Phys. Rep. 153, 1, 1 (1987). DOI: 10.1016/03701573(87)90047-0

[3] Л.Д. Ландау. ЖЭТФ 7, 1, 627 (1937).

[4] Л.Д. Ландау, Е.М. Лифшиц. Статистическая физика. Наука, M. (1976). Ч. 1. 584 c. [L.D. Landau, E.M. Lifshitz. Statistical Physics. Pergamon Press, Oxford, U.K. (1980). V. I.]

[5] Я.И. Френкель. Кинетическая теория жидкостей. Наука, Л. (1975). $592 \mathrm{c}$

[6] С.М. Стишов. УФН 114, 1, 3 (1974). [S.M. Stishov. Sov. Phys. Usp. 17, 5, 625 (1975). DOI: $10.1070 /$ PU1975v017n05ABEH004361

[7] L.B. Robinson, V.S.T. Au-Yeung, H.A. Yassen. Phys. Rev. B 21, 6, 2352 (1980). DOI: 10.1103/PhysRevB.21.2352

[8] Ю.Л. Климонтович. Статистическая физика. Наука, М. (1982). $608 \mathrm{c}$

[9] A. Aitta. J. Status Mech.: Theory Exp. 2006, 12, P12015 (2006). DOI: 10.1088/1742- 5468/2006/12/P12015

[10] J. Kierfeld, V. Vinokur. Phys. Rev. B 61, 22, R14928 (2000). DOI: $10.1103 /$ physrevb.61.r14928

[11] В.В. Прут. ЖТФ 78, 5, 138 (2008). [V.V. Prut. Techn. Phys. 53, 5, 668 (2008).] DOI: 10.1134/S1063784208050241

[12] M. Elenius, M. Dzugutov. J. Chem. Phys. 131, 10, 104502 (2009). DOI: $10.1063 / 1.3213616$

[13] М.Н. Магомедов. Вестн. Московского гос. техн. ун-та им. Н.Э. Баумана. Сер. Естеств. науки 2, 28 (2013).

[14] K. Mochizuki, K. Koga. Phys. Chem. Chem. Phys. 17, 28, 18437 (2015). DOI: $10.1039 / \mathrm{c} 5 \mathrm{cp} 02568 \mathrm{k}$

[15] Д.А. Киржниц. УФН 104, 3, 489 (1971). [D.A. Kirzhnits. Sov. Phys. Usp. 14, 4, 512 (1972).] DOI: 10.1070/PU1972v014n04ABEH004734

[16] S.M. Sharma, S.K. Sikka. Prog. Mater. Sci. 40, 1, 1 (1996). DOI: 10.1016/0079-6425(95)00006-2

[17] G. Shen, H.K. Mao. Rep. Prog. Phys. 80, 1, 016101 (2016). DOI: $10.1088 / 1361-6633 / 80 / 1 / 016101$

[18] С.А. Кукушкин, А.В. Осипов. Неорган. материалы 35, 6, 635 (1999).

[19] M.N. Magomedov. J. Mol. Liq. 285, 106 (2019). DOI: 10.1016/j.molliq.2019.04.032

[20] М.Н. Магомедов. ФТП 42, 10, 1153 (2008) [M.N. Magomedov. Semiconductors 42, 10, 1133-1145 (2008).] DOI: $10.1134 / \mathrm{S} 1063782608100011$

[21] М.Н. Магомедов. Физика металлов и металловедение 114, 3, 227 (2013). [M.N. Magomedov. Phys. Met. Metallography 114, 3, 207 (2013).] DOI: 10.1134/S0031918X13030113

[22] М.Н. Магомедов. ФТП 44, 3, 289 (2010). [M.N. Magomedov. Semiconductors 44, 3, 271 (2010).]

DOI: $10.1134 / \mathrm{S} 1063782610030012$
[23] Дж. Гиршфельдер, Ч. Кертисс, Р. Берд. Молекулярная теория газов и жидкостей. Изд-во И. Л., М. (1961). 931 с. [J.O. Hirschfelder, Ch.F. Curtiss, B.B. Bird. Molecular Theory of Gases and Liquids. J. Wiley and Sons, N.-Y. (1954).].

[24] М.Н. Магомедов. ФТТ 45, 1, 33 (2003). [M.N. Magomedov. Phys. Solid State 45, 1, 32 (2003).] DOI: 10.1134/1.1537405

[25] Л. Жирифалько. Статистическая физика твердого тела. Mup, M. (1975). 383 c. [L.A. Girifalco. Statistical Physics of Materials. J. Wiley and Sons Ltd., N.-Y. (1973).]

[26] С.М. Стишов. УФН 154, 1, 93 (1988). [S.M. Stishov, Sov. Phys. Usp. 31, 1, 52-67 (1988).] DOI: 10.1070/PU1988v031n01ABEH002535

[27] М.Н. Магомедов. Теплофизика высоких температур 44, 4, 518 (2006). [M.N. Magomedov. High Temperature 44, 4, 513 (2006).] DOI: 10.1007/s10740-006-0064-5

[28] N.H. March, N.P. Tosi. Introduction to Liquid State Physics. World Scientific Publishing Co. Pte. Ltd., London (2002). $432 \mathrm{p}$.

[29] G.J. Mikolaj, C.J. Pings. J. Chem. Phys. 46, 4, 1401 (1967). DOI: $10.1063 / 1.1840864$

[30] M. Hanifpour, N. Francois, V. Robins, A. Kingston, S.V. Allaei, M. Saadatfar. Phys. Rev. E 91, 6, 062202 (2015). DOI: 10.1103/PhysRevE.91.062202

[31] M.N. Magomedov. J. Non-Cryst. Solids 546, 120263 (2020). DOI: 10.1016/j.jnoncrysol.2020.120263

[32] М.Н. Магомедов. Кристаллография 62, 3, 487 (2017). [M.N. Magomedov. Crystallogr. Rep. 62, 3, 480 (2017).] DOI: $10.1134 /$ S1063774517030142

[33] G.J. Zarragoicoechea, V.A. Kuz. Phys. Rev. E 65, 2, 021110 (2002). DOI: 10.1103/PhysRevE.65.021110

[34] A.W. Islam, T.W. Patzek, A.Y. Sun. J. Nature Gas.Sci. Eng. 25, 134 (2015). DOI: 10.1016/j.jngse.2015.04.035

Редактор Ю.Э. Китаев 